\title{
Precise and Dynamic Temperature Control in High-Resolution Microscopy with VAHEAT
}

\author{
Jaroslav Icha, Daniel Böning, and Pierre Türschmann*
}

Interherence GmbH, Henkestrasse 91, D-91052 Erlangen, Germany

*pierre@interherence.com

\begin{abstract}
Temperature is a key parameter in most biological and physical systems. It is, however, an inherent technological challenge to precisely track and control the temperature of small sample volumes when using high- and super-resolution microscopes. Various effects can cause a deviation of the sample temperature from its expected value, specifically in the observed volume or field-ofview. These effects include direct thermal contact of the objective lens and the coverslip via immersion medium, illumination-induced heating, evaporative cooling, or triggered phase transitions. In this paper, we shed light on these phenomena and introduce our newly developed device VAHEAT, which follows a microscopic approach for controlling and measuring the sample temperature. Our system overcomes traditional limitations of temperature sensing and controlling in high-sensitivity microscopy applications, leading to more reproducible experimental results and conquering new experimental parameter ranges.
\end{abstract}

Keywords: temperature control, live cell imaging, stage-top incubator, temperature sensing, VAHEAT

\section{Introduction}

Many biological systems require physiological conditions in order to behave naturally or simply to survive during the experiment. Important environmental parameters include salt concentration, $\mathrm{pH}$, atmosphere, and temperature. In solution, salt concentration and $\mathrm{pH}$ can be easily adjusted and maintained on a macroscopic level either using perfusion or robust buffer systems. Atmospheric conditions can be set by changing the composition of the surrounding air with an incubation system. Temperature control, however, is a challenging task, especially when working with high-resolution optical microscopes. The main reason for this is the sample volume, which typically ranges from a few to hundreds of microliters. The associated heat capacity $(10-500 \mathrm{~mJ} / \mathrm{K})$ is four to five orders of magnitude smaller than the heat capacity of surrounding elements such as the microscope objective (approximately $5-15 \times 10^{4} \mathrm{~mJ} / \mathrm{K}$ ). The temperature of the sample volume is accordingly prone to every slight change in net energy input or output (for example, illumination-induced heating), any thermal link to its surroundings (for example, objective lens), or internal energy-releasing or consuming processes (for example, phase transitions).

An often observed but unwanted change of temperature within the field-of-view (FOV) occurs when using high numerical aperture objectives that require an immersion medium. In this configuration, the immersion medium establishes a direct thermal link between the objective and the cover slip. The FOV, thus, adopts a temperature close to the objective's (see Figures 1a and 1b). The precise temperature is, however, an unknown even when working in a thermally stabilized enclosure or incubator, as it depends on many experimental variables including the sample volume, the imaging modalities, and the type of immersion medium. Additionally, other effects such as laser-induced heating, evaporation, or exchange of medium can also lead to a change of temperature by several degrees Celsius. This huge parameter space makes it nearly impossible to have precise knowledge about the temperature in the FOV without measuring and actively controlling it.

Conventional sample heating approaches build up on the belief that macroscopic heating of the entire microscope setup, or at least of the objective in combination with the stage insert, will ensure a certain temperature in the sample volume. These systems suffer, however, from slow equilibration, insufficient temperature precision, limited temperature range, and bulky design. Furthermore, the large amount of heat transferred to the microscope can lead to mechanical drift and limits the range of applications. Prior calibration with an external temperature probe is often necessary, as the built-in temperature probes sense close to, but not within, the sample volume. It turns out that even external calibration with a separate probe is challenging because most temperature probes that are available on the market exhibit similar heat capacities (typically $10-30 \mathrm{~mJ} / \mathrm{K}$ ) as the sample volume. Inserting an external probe into the sample volume can thus substantially change the overall heat capacity and affect the read-out temperature (neglecting the additional thermal link of the electrical wires and sensing current). Only a temperature sensor with negligible heat capacity and good thermal insulation from the surrounding environment would be capable of measuring the correct and unbiased sample temperature.

Here, we present the VAHEAT device, which is based on microscope cover slips that incorporate an optically transparent thin-film heating element made of indium tin oxide (ITO) and a microfabricated platinum temperature probe (Smart Substrates). The probe is less than $100 \mathrm{~nm}$ thick and adds a heat capacity of less than $130 \mathrm{~nJ} / \mathrm{K}$, which is more than five orders of magnitude smaller than the typical heat capacity of a sample volume. Mounting of samples directly onto these cover slips restricts heating to the sample volume and thermally decouples it from the environment. This local heating and temperature sensing enables fast and precise temperature changes with heating rates of up to $100^{\circ} \mathrm{C} / \mathrm{s}$, precision higher than $0.1^{\circ} \mathrm{C}$, and the possibility to program arbitrary temperature profiles similar to PCR thermocyclers. The fast feedback-driven thermal response of the cover slips will counteract any external disturbances introduced by, for example, perfusion systems or changes in the room temperature. Very localized heating also reduces the overall heat load transferred into the setup and leads to tolerable warming up of the objective (Figures 1c and 1d) and minimal thermal drift. Overall, this technology has the potential to enable new types of temperature-sensitive experiments and to help increase the accuracy and reproducibility of imaging studies. 


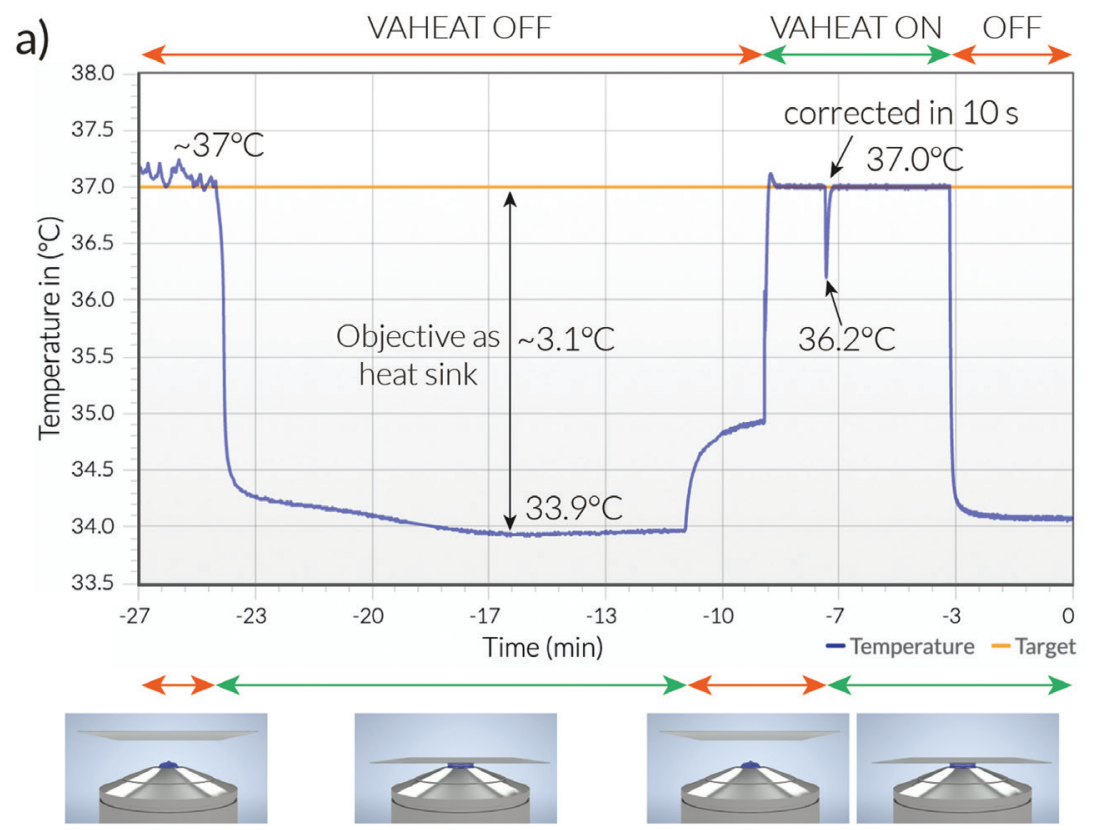

c)

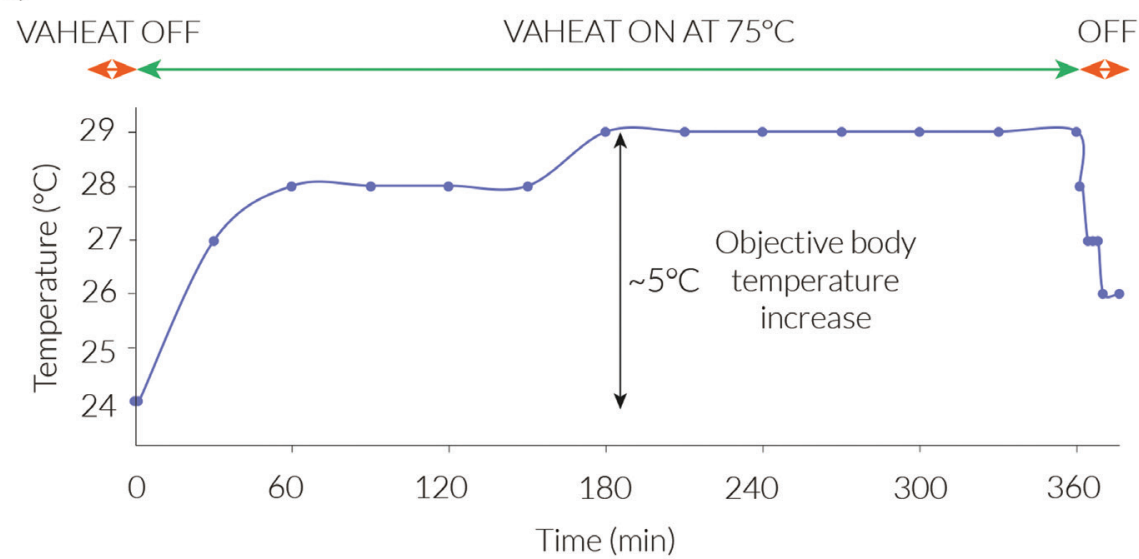

b)
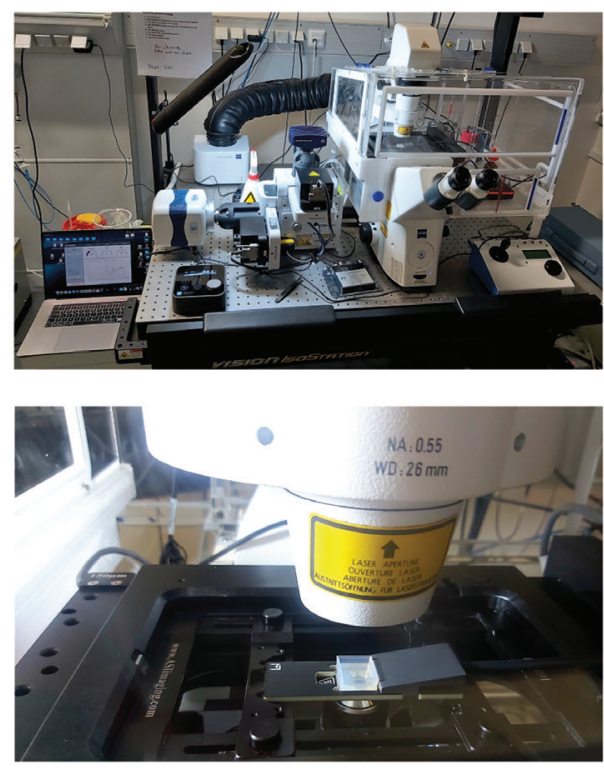

d)

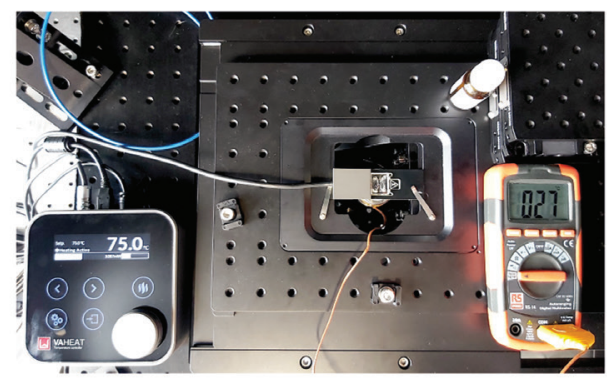

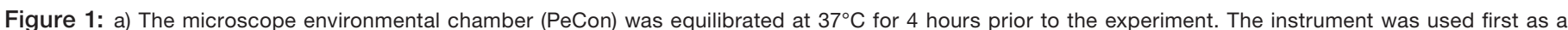

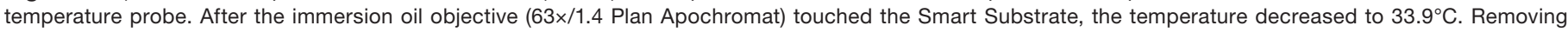

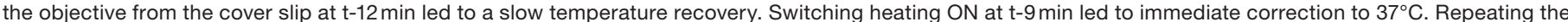

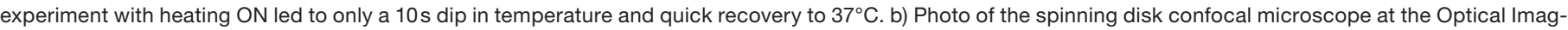

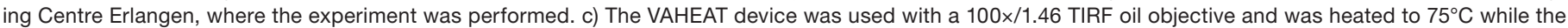

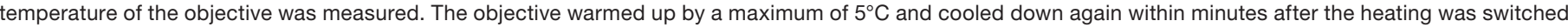
off. d) Photo of the experimental setup with external temperature probe measuring the temperature of the objective body.

\section{Methods and Materials}

Components. The device is comprised of four components, namely (1) the Smart Substrate, (2) the microscope adapter, (3) the probe head, and (4) the control unit (Figure 2a). The core piece is the Smart Substrate, an exchangeable part, that incorporates a precise four-point temperature probe and a transparent, thin-film heating element.

Smart Substrates. The Smart Substrates are designed to replace conventional cover slips in the experimental workflow and are made of borosilicate glass with ultra-low autofluorescence. With the dimensions of $18 \times 18 \mathrm{~mm}$ and a thickness of $170+/-5 \mu \mathrm{m}(\# 1.5 \mathrm{H}$ standard), they are designed for highresolution imaging (Figure 3a). The temperature probe is made of platinum and is less than $100 \mathrm{~nm}$ thick. It is attached to the upper side of the substrate and covered by a thin layer of glass, such that it is in direct thermal contact with the sample but electrically isolated. The transparent heating element is made of a less than $50 \mathrm{~nm}$ thick layer of ITO applied on the lower side. The ITO layer is $5 \times 5 \mathrm{~mm}$ large to ensure a homogeneously heated sample volume in the FOV, even when an oil-immersion objective is used for imaging (Figure 3b). The Smart Substrates are supplied in three different versions: standard range up to $100^{\circ} \mathrm{C}$, extended range up to $200^{\circ} \mathrm{C}$, and a version with reservoirs for liquid samples (Figure 3c). With the possibility to attach custom-made microfluidics or other poly(dimethylsiloxane) (PDMS) reservoirs (Figure 3d), the 
a)

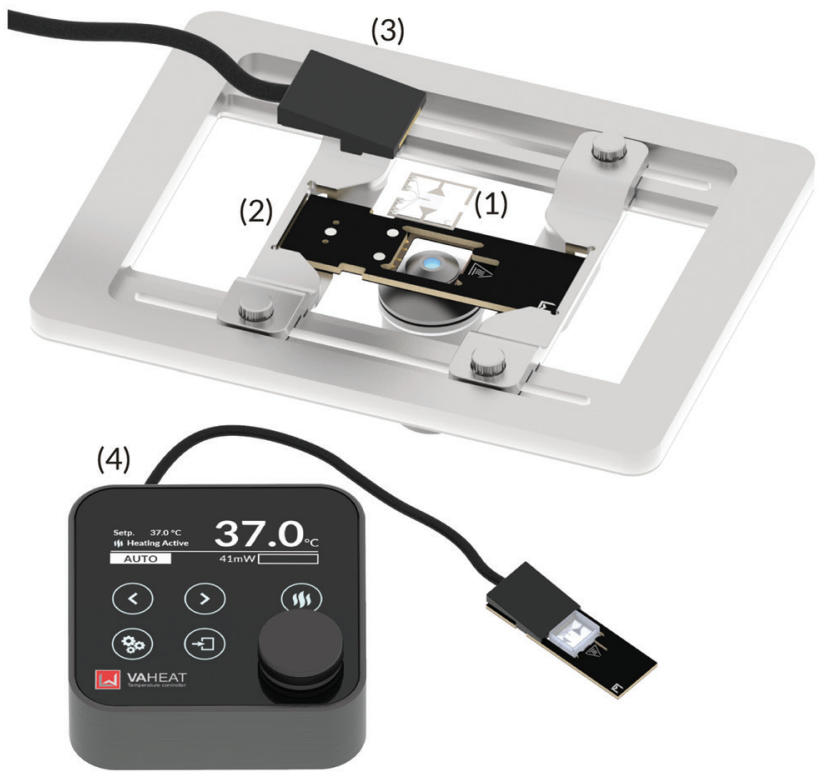

b)

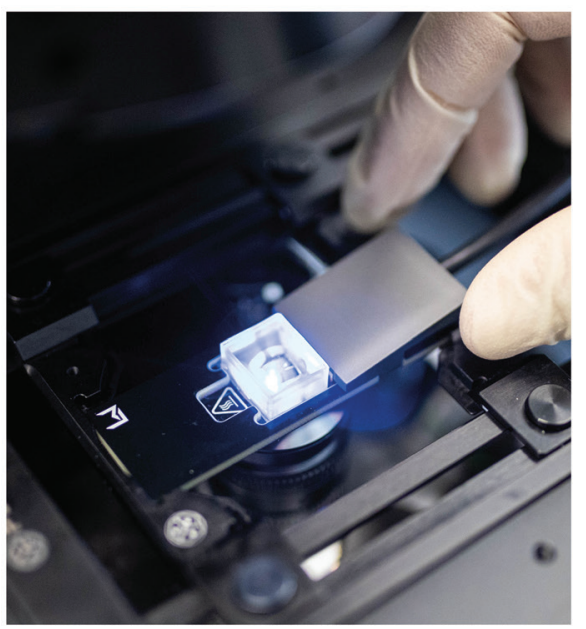

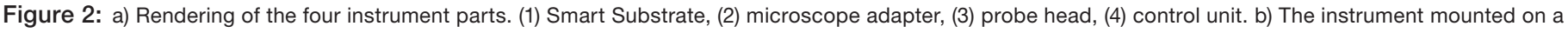
microscope. The version of the Smart Substrate with reservoir for liquid samples is used.

prototypes demonstrate the capability to promptly change experimental design using rapid prototyping and PDMS.

In an inverted microscope, the samples are imaged through the ITO layer (Figure 2b), which has a transmittance in the visible spectrum comparable to standard cover slips, resulting in a negligible impact on the imaging quality. The temperature probe within the smart substrate measures the temperature 80 times per second and is connected through a proportional-integral-derivative (PID) controller to the heating element. This feedback allows compensation for slow, as well as fast, external temperature variation induced by, for example, air flow or fluid exchange. The temperature response of each substrate is characterized after manufacturing, and the resulting two substrate calibration parameters, R25 and TKR, are supplied to the user, who must enter them before the measurement for high-precision experiments. The Smart Substrates can be single-use but can be also washed by most of the conventional organic and water-based solvents and cleaned by sonication. The substrates withstand oxygen plasma cleaning (also applicable for bonding PDMS-based microfluidics) as well as thermal and UV sterilization methods but should not be autoclaved or exposed to low $\mathrm{pH}$.

Microscope adapter. The microscope adapter supports the Smart Substrates and electrically connects the heating element. Its footprint of $75 \times 25 \mathrm{~mm}^{2}$ and height of approximately $2 \mathrm{~mm}$ resembles a standard microscope slide, ensuring broad compatibility with various microscope stages. It is best fixed on microscope stages with metal clips. It can be cleaned using the same protocols as for the Smart Substrates. The opening on the bottom side of the adapter, with a size of $16 \times 16 \mathrm{~mm}^{2}$, ensures unhindered optical access with high numerical aperture objectives.

Probe head. The probe head connects via a magnetic clamp mechanism to the microscope adapter. It holds the
Smart Substrates in place and ensures electrical connection of the substrates and adapter to the control unit. It is designed to allow optical access from the top when working with upright microscopes or applying white light illumination in transmission.

Control unit. The control unit allows precise adjustment and read-out of the sample temperature. A computer interface can be used to synchronize temperature data with, for example, camera frames. The integrated turning knob enables realtime temperature control when studying in situ temperature dynamics. The sample temperature is displayed on the controller as soon as the Smart Substrate is positioned in the cavity of the microscope adapter and the probe head is connected. The control unit offers various heating modes, namely "Auto," "Shock," "Direct," and "Profile." The conventional operational mode is "Auto." Here, the temperature of the sample volume is automatically feedback-controlled, compensating for environmental influences. The mode is activated by pressing "heat," while the set-point temperature can be adjusted using the turning knob. An application programming interface (API) is available to enable device control and parameter readout via a USB-interface. Other operating modes enable direct control of the heating power (direct), the ability to send well-defined heat pulses (shock), or to run more complex temperature protocols (profiles).

Sample preparation. Depending on experimental needs, the sample is prepared on the substrate either before or after connecting it to the control unit. The latter allows setting of the correct temperature of the Smart Substrate before placing the sample. Cells can be seeded on the Smart Substrate, and they adhere normally, not showing preference for any part of the substrates. As the surface pointing toward the sample is made of pure glass, it can be functionalized or passivated by using established protocols. 
a)

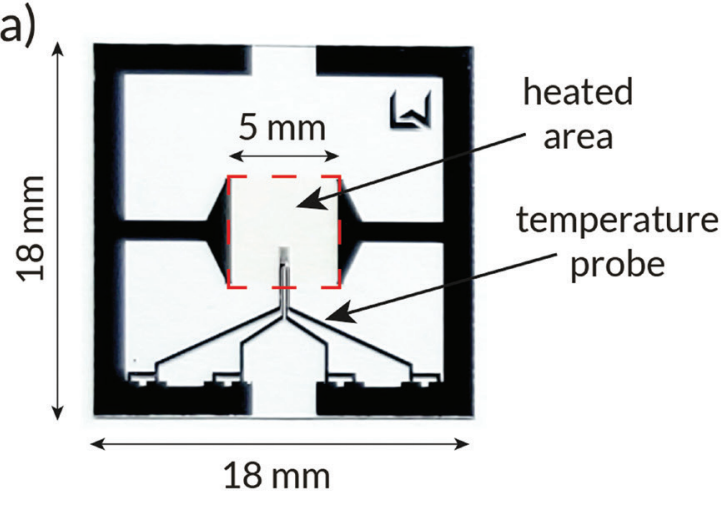

b)

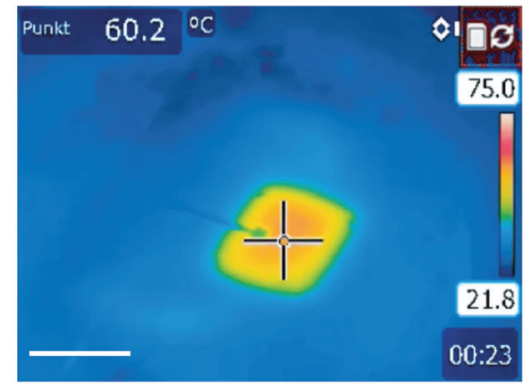

c)

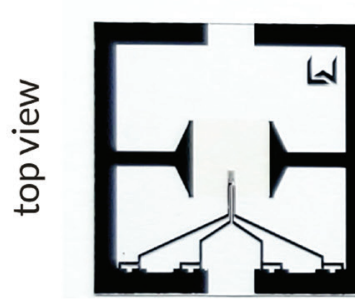

$\frac{3}{\frac{1}{3}}$

d)
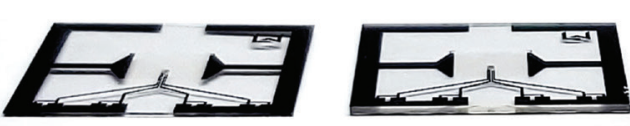

(2)
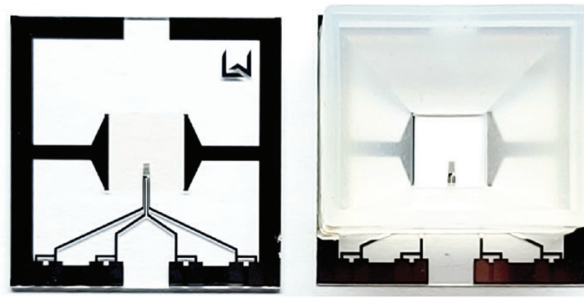

(3)

(4)
(5)

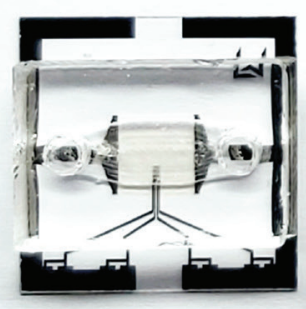

(6)

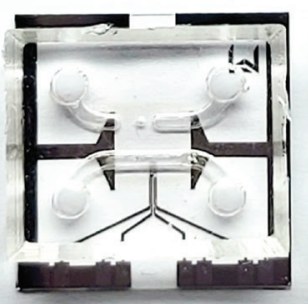

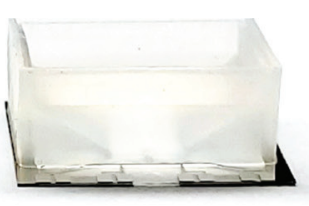

(7)

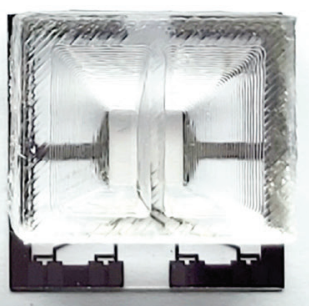

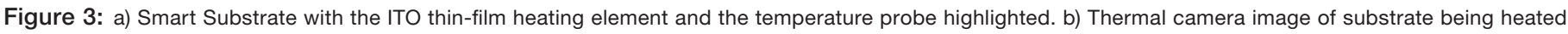

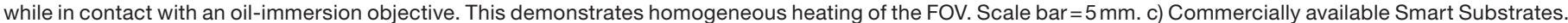

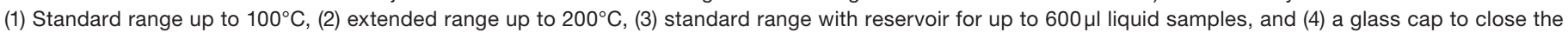

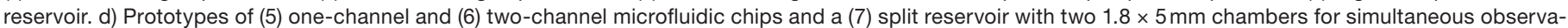
tion of two samples.

\section{Key Features}

Local heating. The heat transferred into the system is kept to a minimum. This reduces thermal drift and tension and preserves the optical imaging quality.

Extended temperature range. Temperature of the sample volume can be tuned between ambient and $200^{\circ} \mathrm{C}$.

Direct sensing. A precise and fast temperature sensor in the FOV measures the temperature 80 times per second. This allows avoidance of external temperature changes coming, for example, from perfusion systems, strong illumination, or air conditioning.

Fast dynamics. Heating rates of up to $100^{\circ} \mathrm{C} / \mathrm{s}$ and direct feedback allow precise temperature stabilization (Figures $4 \mathrm{a}$ and $4 \mathrm{~b})$. Well-defined temperature profiles can be set arbitrarily.

Compatibility. VAHEAT is compatible with all conventional inverted microscopes and many upright setups. There is no need for prior preparation, installation, or calibration. 
The system can even be employed in various incubation or vacuum chambers.

Analog electronics. Both the heating and the sensing elements are all analog devices to minimize electronic noise in the sample volume. This opens the possibility of combining VAHEAT with electronically sensitive measurement schemes such as atomic force microscopy.

\section{Results}

Thermal and optical performance. We experimentally evaluated the temperature step response of the VAHEAT stage under standard operating conditions with no thermal load attached (Figure 4a). Changing the setpoint from room temperature to $37^{\circ} \mathrm{C}$ yielded heating rates of up to $100^{\circ} \mathrm{C} / \mathrm{s}$. This rate is strongly dependent on the thermal load (sample volume, objective, etc.) attached to the Smart Substrates. Typical thermal loads of $400 \mu \mathrm{L}$ of water in combination with an immersion objective result in heating rates of up to $30^{\circ} \mathrm{C} / \mathrm{s}$. The maximal cooling rate depends on the thermal environment but can be as large as $-10^{\circ} \mathrm{C} / \mathrm{s}$. At a typical temperature of $37^{\circ} \mathrm{C}$, the sample temperature can be stabilized down to a two-sigma variation of $0.01^{\circ} \mathrm{C}$ with a sample rate of $20 \mathrm{~Hz}$ (Figure $4 \mathrm{~b}$ ), demonstrating the fast compensation of external thermal effects.

When performing high- or super-resolution microscopy, the optical performance of the imaging system is crucial for the quality of the data obtained. Therefore, the three-dimensional point-spread function (PSF) of $100 \mathrm{~nm}$ fluorescent beads embedded in a gel was measured using a commercial microscope (Nikon Eclipse Ti-2). The temperature of the sample was set between $20^{\circ} \mathrm{C}$ and $100^{\circ} \mathrm{C}$ using the heating stage. Figures $4 \mathrm{c}$ and $4 \mathrm{~d}$ show the measured PSF when imaging with an immersion objective (1.46 NA) and an air objective (0.4 NA). The lateral PSF (x-y plane) stays diffraction-limited up to $80^{\circ} \mathrm{C}$. Above that temperature, the lateral PSF increases most probably due to diffusion of the fluorescent beads. The axial component (z direction) of the PSF exhibits a characteristic elongation with temperature when working with immersion oil (Figure 4c). Here, the effective optical path length changes with increasing temperature because the immersion oil has a non-negligible temperature dependence of its refractive index. This leads to a non-optimized optical path length and deteriorated optical resolution (spherical aberrations). Spherical aberrations at elevated temperatures can be minimized by choosing a special immersion oil or adjusting the correction ring on the objective. An alternative approach at the expense of a lower numerical aperture is to use dry (air) objectives (Figure 4d).

\section{Applications Requiring Precise Temperature Adjustment and Recording}

There are various applications where precise temperature control is essential in optical microscopy. In the following, we discuss representative examples covering various scientific fields.

a) Setup calibration. Determining and controlling the sample temperature in a complex optical setup is not trivial. If absolute temperature reference and/or precision better than $3^{\circ} \mathrm{C}$ is required, separate thermal calibration steps are typically necessary. Besides these static temperature variations, the illumination of a sample can cause local heating due to absorption by the sample or imaging medium (Figures $4 \mathrm{e}$ and $4 \mathrm{f}$ ). Furthermore, triggered phase transitions, mixing enthalpies, or injection of fluid into a microfluidic device can also lead to a change of temperature in the sample volume relative to the global setting. Highly sensitive probes inside the FOV are therefore essential to track and characterize those changes and, if needed, to trigger compensation with the heating element.

b) Live-cell imaging. It is of special importance to ensure a well-defined temperature when working with live cells [1]. A drop or increase in temperature during observation can activate stress responses [2,3], change the properties of the cytoskeleton [4], and generally change cellular processes. Here, the VAHEAT stage can be used to ensure a constant temperature in the sample volume nearly regardless of the environmental conditions during imaging and while manipulating the samples. Figure 5 provides examples of live-cell imaging in mammalian cells at $37^{\circ} \mathrm{C}$ to visualize lysosomes (Figures $5 \mathrm{a}$ and $5 \mathrm{~b}$ ) or cytoskeleton (Figure 5c). Besides static temperature control, VAHEAT can be used to study temperature dependencies of cellular behavior covering a large dynamic range from room temperature up to $100^{\circ} \mathrm{C}$. It is particularly suitable for inducing heat shock and simultaneous imaging of the sample. This approach was used for studying meiosis with temperature-sensitive mutants in yeast [5]. Other examples include calcium imaging in heat-sensitive neurons (unpublished), time-lapse imaging of thermophilic microorganisms [6], and the study of cell biology of Archaea, which is a group of organisms containing many extremophiles that are notoriously hard to image live [7].

c) Phase transitions. Phase transitions are an important subject of research in material and increasingly also in life sciences. Dynamic and precise temperature control is beneficial as it allows one to steer, trigger, and study crystallization or liquid-liquid phase separation in situ. Here, the following topics are of current interest:

Liquid-liquid phase separation of proteins and RNA in cells is speculated to be one of the driving mechanisms behind compartmentalization of cells and maintenance of nonmembranous organelles, such as P-granules in the cytoplasm, and stress granules or compartments in the nucleus, such as nucleoli or sites of active transcription [8]. The appearance or dissolution of these structures is typically sensitive to temperature and is best studied by high-resolution microscopy [9]. Using VAHEAT, the entire phase diagram can be easily obtained.

Biological membranes are composed of many different constituents and exhibit a complex phase diagram depending on composition and environmental conditions. Measuring the dynamics of lipid membranes on a microscopic level is a key component for understanding the behavior of transmembrane proteins, membrane organelles, and ultimately the complex systems such as cells.

Temperature control close to the melting point of organic and inorganic substances plays an important role in material analysis, sample preparation, and quality control. Our device is ideally suited to study, for example, the melting point of micro crystals, polymer films, and the self-assembly and crystallization behavior of colloids [10]. 
a)

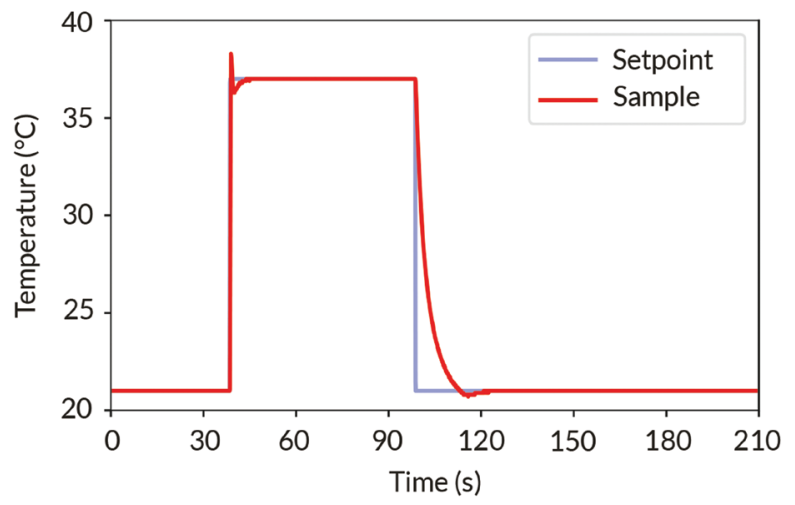

c)
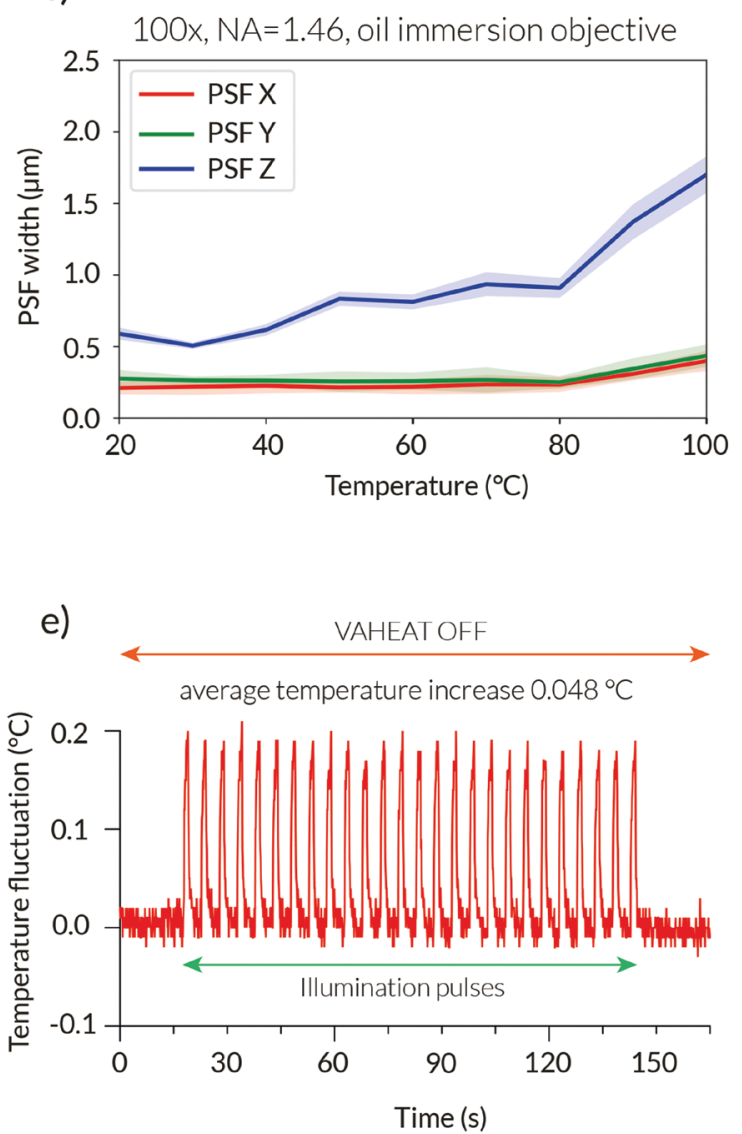

b)

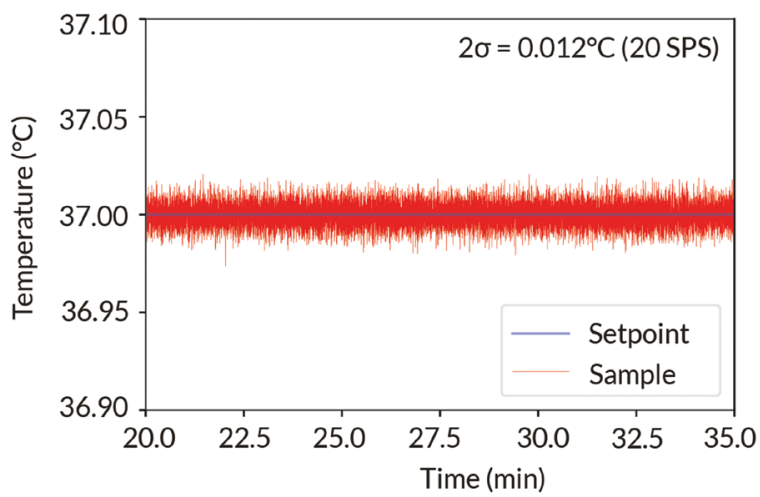

d)
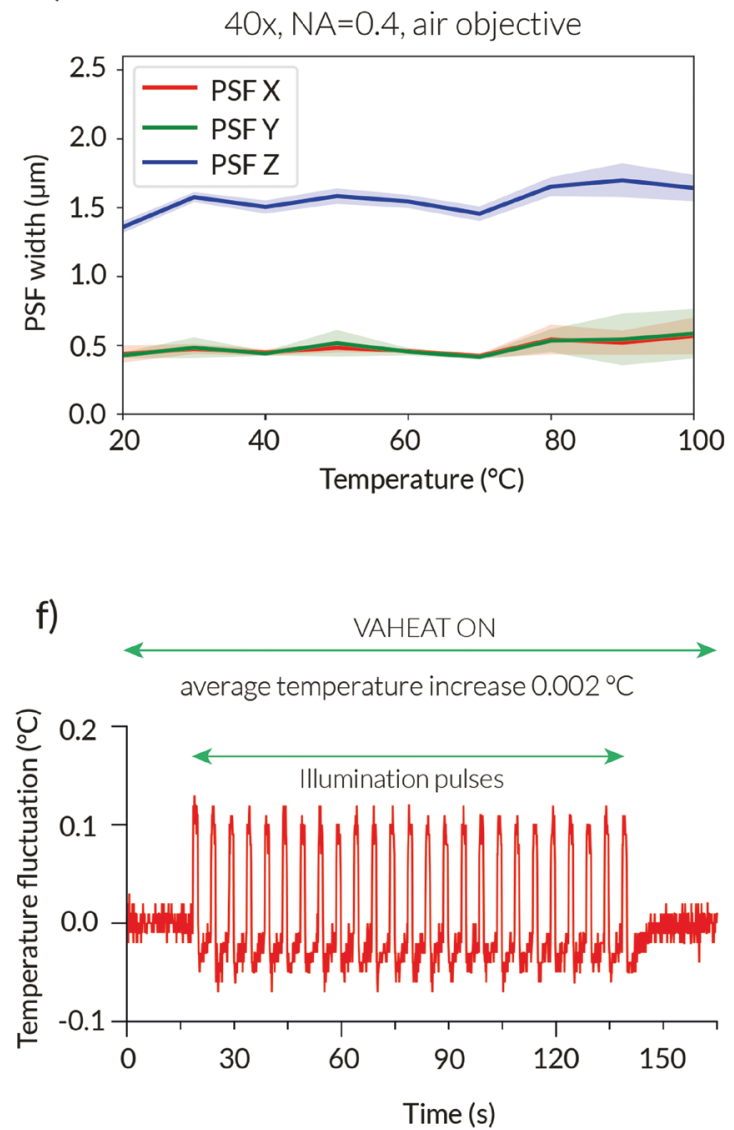

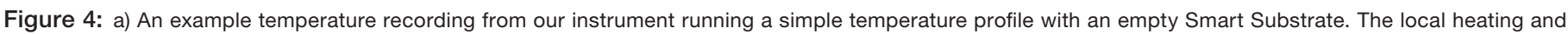

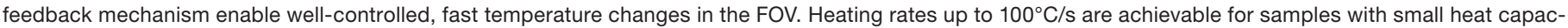

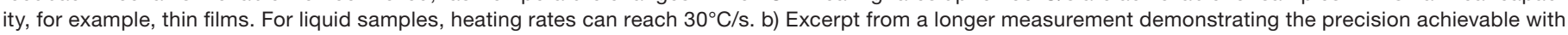

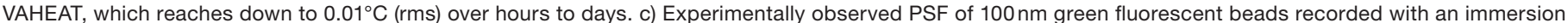

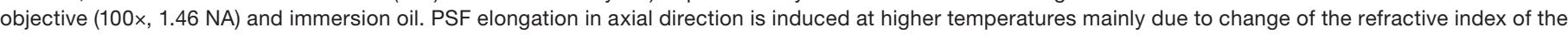

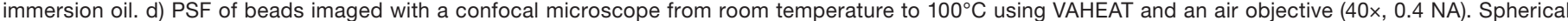

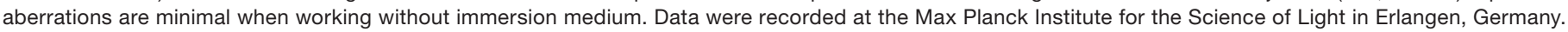

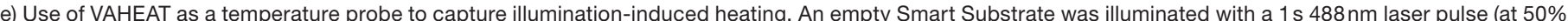

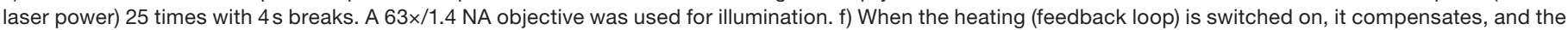
resulting net overheating is close to $0^{\circ} \mathrm{C}$. Experiment was performed in an environmental imaging chamber (PeCon) set to $37^{\circ} \mathrm{C}$ (in e) and $38^{\circ} \mathrm{C}$ (in f).

d) Single-molecule imaging and DNA science. Biological molecules such as proteins are highly sensitive to temperature in their function, interactions, and aggregation [11]. Precise knowledge and control of sample temperature is especially important when tracking single molecules and studying their interactions. In one such study, the VAHEAT device was used to characterize a macromolecular transport system made out of DNA origami [12]. Precise temperature control in 
a) Primary rat cortical neurons

LysoTracker stain (gray)

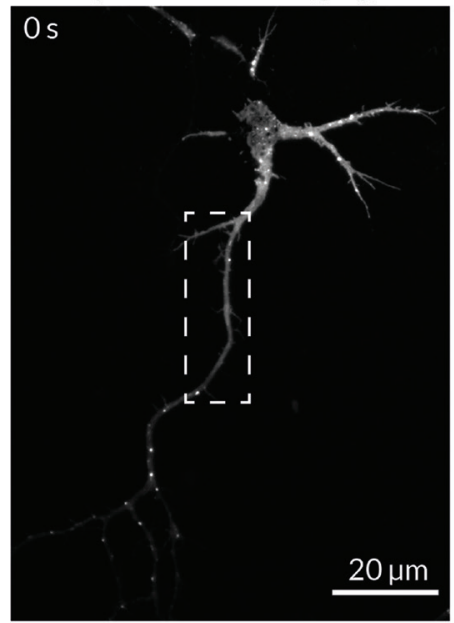

b)

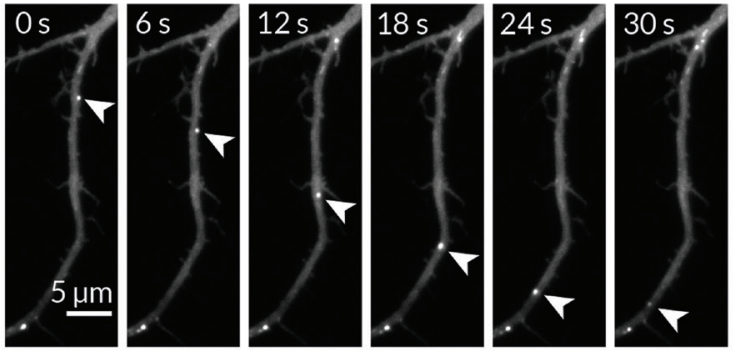

\section{C) Microtubules (SiR-tubulin) in normal dermal fibroblasts}
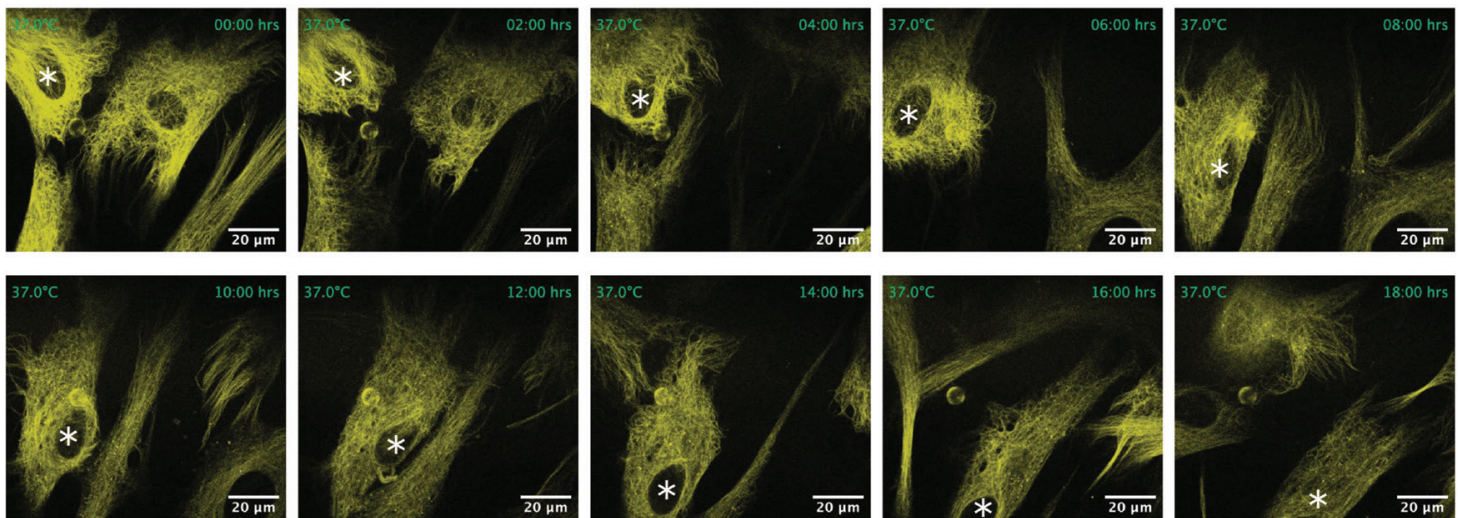

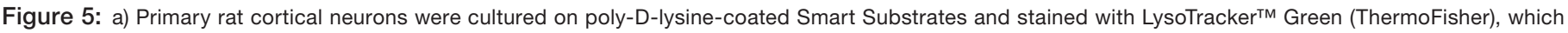

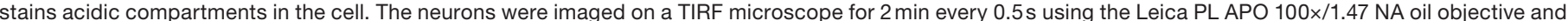

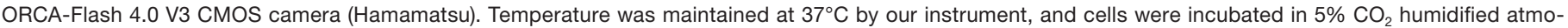

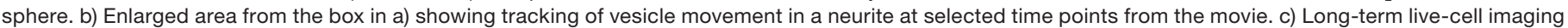

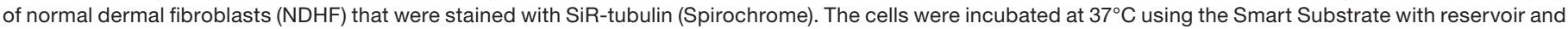
were continuously imaged for more than 18 hours. Images were acquired at the Optical Imaging Centre Erlangen.

these single-molecule TIRF measurements was important for reproducibility.

Hybridization of DNA strands is temperature-sensitive. Typical melting temperatures range between $50^{\circ} \mathrm{C}$ and $90^{\circ} \mathrm{C}$ depending on the number and composition of base pairs. This temperature regime is usually not accessible with conventional heating systems for high-resolution microscopes and opens up new experimental options when imaging DNA-based systems. Especially in context of spatially resolved transcriptomics, temperature-dependent binding and unbinding rates can be exploited to increase the throughput when investigating large samples with methods such as multiplexed error-robust fluorescence in situ hybridization (MERFISH) [13]. Also, in DNA PAINT the dissociation rates are considerably influenced by changes in temperature of only a few degrees Celsius [14].

e) Diffusion processes. Diffusion coefficients strongly depend on temperature. A branch of current research deals with the diffusional behavior of single nanoparticles in complex systems. A well-controlled temperature in the FOV is markedly important in order to extract and model systemrelevant parameters. In a recent preprint, Kashkanova et al. [15] developed a method based on interferometric scattering (iSCAT) microscopy to evaluate complex mixtures of nanoparticles. Our device was used to characterize temperature dependence of diffusion constant of gold nanoparticles.

f) Nanophotonic structures. Temperature control of nanophotonic structures, such as gold nanoparticles or photonic crystals, is useful to tune their resonance frequency and change their shape $[16,17]$. Local, instead of global, temperature control increases the achievable temperature range, improves long-term temperature stability, enables fast resonance tuning, and allows use in vacuum chambers.

\section{Discussion}

Designing a general-purpose temperature control system that is suitable for imaging large (for example, multi-well 
plates) as well as small sample volumes (for example, single wells) is impossible. Macroscopic approaches for heating and sensing the temperature might be suited for some experiments, but only allow for slow heating rates, limited temperature range, and low precision. On the other hand, in high- and super-resolution microscopy, researchers investigate small sample volumes with a distinct local temperature that can substantially deviate from the one set globally. Miniaturizing the sensing and heating circuitry as well as restricting it to the sample volume brings precision, speed, and an extended temperature range.

Although the VAHEAT system follows the miniaturization approach, it still faces minor restrictions in its current implementation. For example, it does not take care of gas or humidity control. However, it can be combined with many commercial or custom-made incubators. The working principle of heating and temperature control is independent of the atmosphere present and also works in vacuum, which is relevant for many single-molecule experiments. Another limitation is that the lid for the reservoirs is not heated. This can result in condensation and thus lead to a disturbed illumination profile in transmission. A simple but powerful strategy to avoid condensation is adding mineral oil on top of the sample volume, an approach common in mammalian embryo cultivation. Another strategy employed by some users is enclosing the sample in a glass capillary placed on the substrate. Yet another method to avoid evaporation and condensation is to use a microfluidic chip, with which the substrates are compatible. However, having no lid as an essential component of the system brings the advantage of easy access for manipulating the sample during experiments.

Some experiments require cooling the samples below ambient temperature. At this point the VAHEAT system does not have active cooling capability, however, due to the relatively small volume that is heated, the passive cooling from maximal back to ambient temperature takes only seconds to minutes depending on the sample volume. To reach below ambient temperature, this device can be combined with a cooled enclosure around the microscope adapter.

Lastly, the heated area on the substrates is limited to $5 \times 5 \mathrm{~mm}^{2}$, which can be small for some large samples and microfluidic chips. This design is, however, motivated by maintaining maximum precision and temperature homogeneity across the entire heated area. It is straightforward to manufacture substrates with a larger heated area, for example, $13 \times 10 \mathrm{~mm}$ for applications that do not require higher than $0.1^{\circ} \mathrm{C}$ absolute precision. Due to the modular design of the device, independent upgrades to the substrates, microscope adapter, probe head, or the control unit can be made in the future. It will be straightforward to accommodate requirements of researchers and new experimental methods.

\section{Conclusions}

Measuring and controlling the temperature of small sample volumes during imaging is non-trivial, as the sample heat capacity is minuscule compared to the capacity of the measurement apparatus. We show that introducing a microscopic temperature probe on a cover slip with negligible heat capacity enables, for the first time, tracking of the sample temperature during experimentation with high precision. Combining this temperature probe with a transparent thin-film heating element (Smart Substrate) results in a potent heating stage, which is easy to use and is well-suited for a large variety of temperature-sensitive imaging studies. The fast feedback loop between temperature sensing and local heating ensures temperature stability better than $0.1^{\circ} \mathrm{C}$ and allows programming of complex temperature profiles similar to a thermal cycler. Using standard cover slips and a microscope adapter ensures compatibility with most of the inverted and many upright light microscopes. This system opens up new possibilities for designing experiments in high-resolution microscopy starting in biology and reaching out to chemistry and material science, wherever precise temperature control is crucial for reproducible and physiologically meaningful results.

\section{Acknowledgements}

We would like to thank the Optical Imaging Center (OICE) of the Friedrich Alexander University Erlangen-Nuremberg (FAU) and the Max Planck Institute for the Science of Light (MPL) in Erlangen for extensive discussions and their help with various experiments.

\section{References}

[1] R Cole, Cell Adhesion Migration 8 (2014) https://doi .org/10.4161/cam.28348.

[2] KS Keuenhof et al., J Cell Sci 135 (2021) https://doi .org/10.1242/jcs.258325.

[3] K Richter et al., Mol Cell 40 (2010) https://doi.org/10.1016/ j.molcel.2010.10.006.

[4] YW Chiou et al., PLoS One 8 (2013) https://doi.org/10.1371/ journal.pone.0077384.

[5] V Mengoli et al., EMBO J 40 (2021) https://doi.org/10 $.15252 / \mathrm{embj} .2020106812$.

[6] C Molinaro et al., RSC Adv 11 (2021) https://doi .org/10.1039/d1ra00184a.

[7] AW Bisson-Filho et al., Mol Biol Cell 29 (2018) https://doi .org/10.1091/mbc.E17-10-0603.

[8] S Alberti and AA Hyman, Nat Rev Mol Cell Biol 22 (2021) https://doi.org/10.1038/s41580-020-00326-6.

[9] S Alberti et al., Cell 176 (2019) https://doi.org/10.1016/ j.cell.2018.12.035.

[10] WB Rogers et al., Nat Rev Mater 1 (2016) https://doi .org/10.1038/natrevmats.2016.8.

[11] GN Somero, Ann Rev Physiol 57 (1995) https://doi .org/10.1146/annurev.ph.57.030195.000355.

[12] P Stömmer et al., Nat Commun 12 (2021) https://doi .org/10.1038/s41467-021-24675-8.

[13] C Xia et al., Proc Natl Acad Sci USA 116 (2019) https://doi .org/10.1073/pnas.1912459116.

[14] J Stein et al., Nano Lett 19 (2019) https://doi.org/10.1021/ acs.nanolett.9b03546.

[15] AD Kashkanova et al., bioRxiv (2021) https://doi .org/10.1101/2021.11.13.468485.

[16] G Xu et al., J Appl Phys 104 (2008) https://doi .org/10.1063/1.2973341.

[17] B Wild et al., Appl Phys Lett 84 (2004) https://doi .org/10.1063/1.1645675. 